Neurosurg Focus 4 (6):Article 9, 1998

\title{
Bias, benefit, or both: evaluating new glioma therapies
}

\section{James R. Perry, B.Sc., M.D., F.R.C.P.(C)}

Division of Neurology, Department of Medicine, Sunnybrook Health Science Centre and the University of Toronto, Toronto, Ontario, Canada

Despite the development of many new promising therapies for malignant glioma, virtually all randomized controlled trials testing them have proven negative. These disappointing results are largely due to complex mechanisms of treatment resistance, but increasingly there is evidence that experimental bias rather than benefit accounts for both the promising early phase I/II trial results and later phase III failures. This paper highlights the aspects of clinical trial design and outcome analysis that specifically affect interpretation of results from therapeutic trials for malignant glioma. Phase II trials of both tumor response and tumor control are subject to selection bias; the early promising results seen with interstitial brachytherapy and intraarterial chemotherapy and yet negative phase III results are examples of this. Methods for detecting selection bias include modeling techniques in which databases of patients with known outcomes are used to emulate phase III outcomes. Modeling may assist in the determination of whether a given phase II result appears to exceed that expected by selection bias alone. Such an experiment on paper is quite unlikely to replace a well-designed randomized trial; however, in this time of increasing numbers of novel therapies but shrinking resources, these techniques should find utility in selecting those therapies most suitable for testing in cooperative group randomized trials.

\section{Key Words * brain neoplasms * clinical trial * bias * phase II study}

In 1978 the Brain Tumor Study Group reported that surgery and radiotherapy were a better treatment protocol for malignant glioma than surgery alone.[16] Unfortunately, in the 20 years since that trial no other treatment added to standard surgery and radiotherapy has unequivocally prolonged tumor control or patient survival when tested in randomized controlled trials. $[5,12]$ These disappointing results in large part reflect a complex and resilient disease; however, inadequate treatment alone does not explain the fact that virtually all therapies found lacking in phase III trials were brought forward based on promising survival data from phase II studies. Several groups have demonstrated that pretreatment prognostic factors influence a patient's outcome more so than the actual treatment given, $[3,6,8]$ and these prognostic factors can be used to define groups of patients destined for a favorable outcome. Examples of promising new therapies found later to be phase III failures include intraarterial chemotherapy,[8] interstitial brachytherapy,[6] and stereotactic radiosurgery.[4,7] In this report these examples will be discussed and methods for the detection and avoidance of similar bias in future studies presented. Outcomes of interest for future trials including survival, quality of life, and patient preference for care will be discussed as an 
addition to general recommendations given as part of a consensus paper presented elsewhere.[12]

\section{DESIGN AND ANALYSIS OF PHASE II TRIAL DATA}

A phase II study is performed when new therapies are identified in the laboratory and dosing and preliminary safety information are known from phase I trials. In general a phase II trial is used to determine in a nonblinded, open-label fashion whether a certain level of disease activity is seen in patients exposed to the therapy. Commonly, a response rate is used as the prime outcome of interest. Like most phase II outcomes, response rate is a binary variable (response, yes or no) and is descriptive in nature (complete, partial, or no response, for example). Pooled response rates from individual patients are usually compared with historical controls, and a variety of well-known stopping rules are usually used to predetermine the sample size required to rule in or rule out a certain level of benefit. Phase II trials are of two types: 1) those that measure tumor response and 2) those that measure tumor control.[11] Investigators use tumor-response trials to attempt to determine the rate and duration of response to a particular therapy; typically this design is used for cytotoxic drugs in which measurable responses may occur. Tumor control trials, however, are used to measure the effects of therapy on progression-free and overall survival; this design is more suited to therapies in which a measurable response may not occur (such as, antiinvasion or antiangiogenesis therapy) or cannot be measured because of local inflammatory effects (such as intracavitary radiation). Phase II trials of both types are important; however, tumor-control trials may predominate as traditional cytotoxic therapies give way to novel therapies that inhibit invasion and limit tumor growth.[12]

\section{Tumor-Response Trials}

Phase II tumor-response studies are currently the major modality used to test therapies for most forms of cancer, and although brain tumors are no exception to this, expected response rates are lower than for most other solid cancers and are on the order of $30 \%$. A noteworthy exception to this, and an instructive example of the utility of response rate measurement, are oligodendrogliomas. Oligodendrogliomas, pure or mixed, may be one of the most chemosensitive of all human solid cancers;[11] in a phase II study Cairncross, et al.,[1] found that recurrent anaplastic oligodendrogliomas respond predictably and durably to the chemotherapy drug combination procarbazine, CCNU (lomustine), and vincristine - PCV. Like other chemosensitive solid malignancies, there is a direct relationship between the degree and duration of response for oligodendroglioma,[2] and this is the main reason that measurement of response rate is an important outcome in phase II trials. Macdonald, et al.,[10] developed standardized response criteria for malignant glioma that, although not yet validated, are used in the vast majority of trials of new therapy (Fig. 1). Unfortunately, a high response rate alone is no guarantee that a drug will later show benefit in phase III trials, and the desirable response rate by which to select drugs for further study is unclear. Moreover, response rate determination is dependent on a number of variables in individual patients; changes in steroid dose, modality of imaging (computerized tomography compared with magnetic resonance imaging), and timing of contrast administration may all be factors that influence the apparent size of disease on a radiological study. Accordingly, there are no definite criteria or cut offs by which to select therapies that warrant evaluation in phase III studies based on response rate alone. Lastly, and perhaps most importantly, response rate per se may not represent the outcome most valued by patients who are likely to value increased disease-free survival and quality of life, rather than response alone. Thus, phase II tumor-response trials, although informative, do not measure outcomes of most interest to patients and clinicians alike, and therapies "advanced" to phase III based on response rate alone may not confer the expected benefits when the outcomes of progression-free survival, overall survival, and 
quality of life are measured.
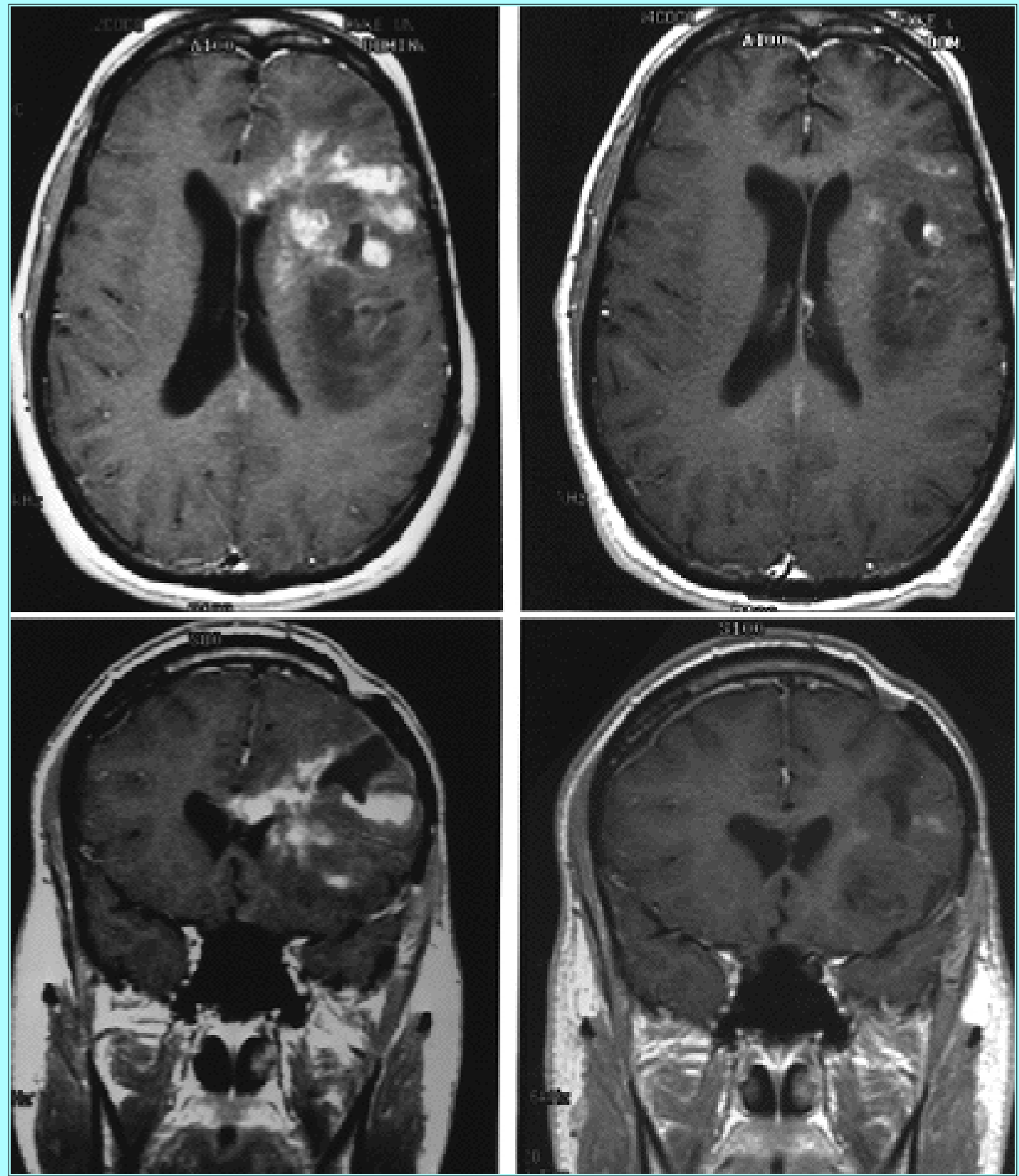

Fig. 1. Gadolinium-enhanced $\mathrm{T}_{1}$-weighted axial (upper) and coronal (lower) views obtained in a 35-year-old man with a pure anaplastic oligodendroglioma before (left) and after (right) two cycles of PCV chemotherapy. A small amount of residual enhancement remains; although dramatic, this is a partial response, and it has been termed a major partial response by some (>90\% reduction in maximum cross-sectional area).

\section{Tumor-Control Trials}

Phase II tumor-control studies are increasingly common in neurooncology for testing therapies such as brachytherapy, intracavitary therapy, stereotactic radiosurgery, and regional treatment such as immunotherapy; in these situations responses cannot be measured because of local inflammatory reaction or because the expected biological effect is not manifest as radiographically evident tumor shrinkage. The chief disadvantage of a tumor-control study is that the outcome measures, including survival, are compared to historical controls and are subject to selection bias and misleading results. For example, 
Florell, et al.,[6] analyzing the survival time in patients with malignant glioma judged eligible for brachytherapy but treated conventionally, have observed that the eligible (but still untreated) patients lived significantly longer than ineligible patients. Patients eligible for brachytherapy survived a median of 16.6 months compared with 9.3 months for ineligible patients; in retrospect these longer-surviving treatment-eligible patients had favorable pretreatment prognostic factors, and the survival benefit could be entirely explained on the basis of selection bias. The later finding by the Brain Tumor Cooperative Group that brachytherapy had little impact on patient survival times in a phase III trial[15] was perhaps not surprising in light of these observations. Subsequently, Curran, et al.,[3] reanalyzed a pooled database from several Radiation Therapy Oncology Group (RTOG) studies and found that the variables of patient age, tumor grade, postoperative performance status, duration of symptoms, and present neurological signs defined six subsets of patients each with a distinct prognosis. Each subset of patients underwent similar treatment in the context of controlled trials, yet their median survival times ranged from 4.7 to 58.6 months. Again, pretreatment prognostic variables outweighed the effects of therapy itself; this may suggest that, barring dramatic effects on patient survival time, these variables, unless accounted for, will overshadow the abilty to detect the true therapeutic benefit of new treatments in clinical trials.

\section{Selection Bias}

Patient selection bias has also been shown to occur in other treatment modalities including intraarterial chemotherapy and stereotactic radiosurgery. Kirby and colleagues,[8] used a database of conventionally treated patients to model treatment outcomes for patients judged eligible for intraarterial BCNU (carmustine); patient selection for a phase III trial was emulated. Intraarterial BCNU showed promising phase II results but was found to be ineffective and rather toxic in a multicenter phase III study.[14] Kirby, et al.,[8] found similar survival times for both eligible and ineligible patients and concluded that patient selection bias accounted for the apparent benefits of such treatment.

Stereotactic radiosurgery is a relatively new technique in which focused radiotherapy is used in patients with small tumors technically suited to treatment planning. Analysis of early data from a study by Loeffler, et al.,[9] suggested a median survival of 19.7 months in patients with newly diagnosed glioblastoma multiforme treated with radiosurgery. Three groups have now analyzed selection bias for radiosurgery based on the premise that the eligibility criteria for the treatment may select out a good prognosis group of patients, making the survival data presented by Loeffler and coworkers, considerably less striking. Using the RTOG database Curran, et al.,[4] found that eligible patients lived longer than ineligible patients (12.5 compared with 10.5 months, respectively); these authors concluded that selection bias did not explain all of the variability needed to account for the long survival time noted by Loeffler, et al., and called for a randomized trial of radiosurgery. Sarkaria, et al.,[13] used a database modeling technique matching pretreatment prognostic variables for ineligible and eligible patients; however, as pointed out by Irish, et al.,[7] they were unable to use information on tumor size (an important eligibility criterion) because their database did not contain this information. They found that eligible patients lived longer than expected but, like Curran, et al.,[4] concluded that a controlled trial was needed. In a third study, the database of Florell, et al.,[6] was used to answer the same question.[7] The advantage of this population-based database approach is the availability of imaging information and actual films so that treatment selection can be accurately simulated and evaluated, including blinded assessments of eligibility. Eligible patients were found to have a median survival of 16.4 versus 8.8 months for ineligible patients leading Irish, et al.,[7] to conclude that selection bias accounts for the long survival times observed in preliminary studies and that a randomized trial was unlikely to reveal benefit from treatment. 
Based on the data from the first two studies, the RTOG initiated a randomized trial that, in addition to answering the question of benefit from radiosurgery, may also help to answer which modeling technique best approximates the results of a randomized trial.[12]

\section{Modeling Techniques}

Modeling techniques to analyze phase II data are attractive and simple to use; however, several limitations exist. First, one must be aware of all of the significant prognostic variables affecting the outcome of patients. Second, one can model only available data; in the preceeding example Sarkaria, et al.,[13] were unable to incorporate tumor size based on pretreatment imaging into their analysis. Third, only certain outcomes can be modeled; survival data are usually available, but other outcomes of interest such as quality of life and patient preferences are usually not. Modeling could not be used to predict an outcome by which survival was increased only slightly but quality of life was improved substantially. Fourth, population-based databases may have regional specificity associated with them and not be generalizable to other geographically or culturally disparate centers. Lastly, the utility and validity of modeling techniques have not been evaluated and are at best a tool by which outcomes may be estimated. These techniques are unlikely to replace randomized trials but may be used as one means by which phase II data can be analyzed and promising therapies selected for promotion to phase III trials. It has been suggested that a phase III trial should be mounted only when investigators can demonstrate that progression-free or total survival exceeds that of controls over and above any differences due to selection bias.[12]

For many years randomized controlled trials of malignant glioma therapy have been disappointing, and this has caused the neurooncology community to pay more attention to the issue of patient selection, bias, and outcomes in malignant glioma research. Bias mistaken for efficacy has led to costly, negative phase III trials that have largely ignored endpoints such as quality of life, cost, and patient preference. Analysis of the 20 years of randomized trials for malignant glioma therapy now provides a good framework for the analysis of novel new therapies; using these newer techniques, the chance of finding agents with efficacy will be increased and hopefully bring an end to these two decades of frustrated learning.

\section{References}

1. Cairncross G, Macdonald D, Ludwin S, et al: Chemotherapy for anaplastic oligodendroglioma. J Clin Oncol 12:2013-2021, 1994

2. Cairncross JG, Eisenhauer EA: Response and control: lessons from oligodendroglioma. J Clin Oncol 13:2475-2476, 1995 (Letter)

3. Curran WJ Jr, Scott CB, Horton J, et al: Recursive partitioning analysis of prognostic factors in three Radiation Therapy Oncology Group malignant glioma trials. J Natl Cancer Inst 85:704-710, 1993

4. Curran WJ Jr, Scott CB, Weinstein AS, et al: Survival comparison of radiosurgery-eligible and -ineligible malignant glioma patients treated with hyperfractionated radiation therapy and carmustine: a report of Radiation Therapy Oncology Group 83-02. J Clin Oncol 11:857-862, 1993

5. Fine HA: The basis for current treatment recommendations for malignant gliomas. J Neurooncol 20:111-120, 1994

6. Florell RC, Macdonald DR, Irish WD, et al: Selection bias, survival, and brachytherapy for glioma. J 
Neurosurg 76:179-183, 1992

7. Irish WD, Macdonald DR, Cairncross JG: Measuring bias in uncontrolled brain tumor trials--to randomize or not to randomize? Can J Neurol Sci 24:307-312, 1997

8. Kirby S, Brothers M, Irish W, et al: Evaluating glioma therapies: modeling treatments and predicting outcomes. J Natl Cancer Inst 87:1884-1888, 1995

9. Loeffler JS, Alexander E III, Shea WM, et al: Radiosurgery as part of the initial management of patients with malignant gliomas. J Clin Oncol 10:1379-1385, 1992

10. Macdonald DR, Cascino TL, Schold SC Jr, et al: Response criteria for phase II studies of supratentorial malignant glioma. J Clin Oncol 8:1277-1280, 1990

11. Perry JR, Cairncross JG: Oligodendrogliomas, in Vecht CJ (ed): Handbook of Clinical Neurology, Vol 24. Amsterdam: Elsevier, 1998

12. Perry JR, DeAngelis LM, Schold SC Jr, et al: Challenges in the design and conduct of phase III brain tumor therapy trials. Neurology 49:912-917, 1997

13. Sarkaria JN, Mehta MP, Loeffler JS, et al: Radiosurgery in the initial management of malignant gliomas: survival comparison with the RTOG recursive partitioning analysis. Int J Radiat Oncol Biol Phys 32:931-941, 1995

14. Shapiro WR, Green SB, Burger PC, et al: A randomized comparison of intra-arterial versus intravenous BCNU, with or without intravenous 5-fluorouracil, for newly diagnosed patients with malignant glioma. J Neurosurg 76:772-781, 1992

15. Shapiro WR, Green SB, Burger PC, et al: A randomized trial of interstitial radiotherapy (IRT) boost for the treatment of newly diagnosed malignant glioma (glioblastoma multiforme, anaplastic astrocytoma, anaplastic oligodendroglioma, malignant mixed glioma): BTCG study 8701. Neurology 44 (Suppl 2):A263, 1994 (Abstract)

16. Walker MD, Alexander E Jr, Hunt WE, et al: Evaluation of BCNU and/or radiotherapy in the treatment of anaplastic gliomas. A cooperative clinical trial. J Neurosurg 49:333-343, 1978

Manuscript received April 20, 1998.

Accepted in final form May 20, 1998.

Address reprint requests to: James R. Perry, M.D., Room A-442, Division of Neurology, Sunnybrook Health Science Centre, 2075 Bayview Avenue, Toronto, Ontario, Canada, M4N 3M5. email: jperry@srcl.sunnybrook.utoronto.ca. 